\title{
Minimum Cost Spanning Tree Problems with Indifferent Agents
}

\author{
Christian Trudeau (University of Windsor)
}

Working paper $13-06$

Working papers are in draft form. This working paper is distributed for purposes of comment and discussion only. It may not be reproduced without permission of the copyright holder. Copies of working papers are available from the author or at http://ideas.repec.org/s/wis/wpaper.html. 


\title{
Minimum cost spanning tree problems with indifferent agents
}

\author{
Christian Trudeau \\ Economics Department, University of Windsor
}

November 5, 2013

\begin{abstract}
We consider an extension of minimum cost spanning tree (mcst) problems where some agents do not need to be connected to the source, but might reduce the cost of others to do so. Even if the cost usually cannot be computed in polynomial time, we extend the characterization of the Kar solution (Kar (2002, GEB)) for classic mcst problems. It is obtained by adapting the Equal treatment property: if the cost of the edge between two agents changes, their cost shares are affected in the same manner if they have the same demand. If not, their changes are proportional to each other. We obtain three variations on the Kar solution, that are differentiated and characterized using stability, fairness and manipulation-proofness properties.
\end{abstract}

JEL Classification: C71, D63

Keywords: Minimum cost spanning tree; Steiner tree; cost sharing; Shapley value

Minimum cost spanning tree (mcst) problems study situations in which a group of agents need to connect to a source in order to obtain a good or service. There is a cost to be paid for each edge in the network that is used. That cost is independent of the number of agents that use that edge to connect to the source. There is a large literature on the associated cost-sharing problem, with the first use of game-theoretic tools appearing in Bird (1976). That article showed that the core is always non-empty and proposed a method, now known as the Bird solution, that is always in the core.

We propose an extension to the mcst model: instead of assuming that all agents desire to be connected to the source, we allow for a subset of agents to be indifferent to such a connection. While these agents have no demand, they still may have an impact on the cost of the project as their cooperation might allow other agents to connect to the source at a cheaper cost. We then have the non-trivial problem of potentially compensating these agents for their contributions to lowering cost.

This extension allows us to cover a wide range of problems. Obviously, if all agents want to be connected, we are back in the classic mest problem. If only one agent wants to be connected, this turns out to be equivalent to a shortest path problem. The problem considered is also close to Steiner tree problems (see Hwang and Richards (1992) for a review), in which some freely available nodes can be used by anyone. Steiner tree problems and the general problem considered here share the undesirable characteristics that the minimum cost of the project usually cannot be computed in polynomial time and that the core can be empty. Bergantinos et al. (2011) attempts to provide cost-sharing solutions to Steiner tree problems. The difference with our setup is that we do not consider these nodes as unoccupied, and we allow for compensation to their owners. If such compensations were disallowed, the problem would become a Steiner tree problem. ${ }^{1}$

The relevance of the extension is not only theoretical: the minimum cost spanning tree problem applies well to physical distribution networks of goods like water, gas or electricity. However, most of these projects involve states or cities that do not want the good (or are not its main destination) but

\footnotetext{
${ }^{1}$ In Trudeau (2012), there is a distinction between the common and the private property approaches. In the former, a coalition can use the location of any of its neighbours to connect to the source, while in the latter the coalition can only use the locations of its members.

Using the common property approach with indifferent agents would be equivalent to disallowing compensations to indifferent agents. Throughout the paper, we use the private property approach.
} 
that host part of the network. Conflicts with these agents are frequent, as the use of their territory usually requires compensation. ${ }^{2}$

The cooperation among agents that need to be connected to the source and agents that can provide them with cheaper connections shares similarities with the general technological cooperation models (Trudeau (2009a), Bahel and Trudeau (2013)) in which agents provide an unspecified service (sharing patents, know-how, providing access to suppliers, etc.) that reduces the overall cost. These issues have been raised for the mcst problems, notably when discussing if it is acceptable to subsidize some agents, as the Kar solution does (Bergantinos and Vidal-Puga (2007), Trudeau (2012)).

Three of the main cost sharing solutions in the minimum cost spanning tree literature (the Bird solution, the folk solution (Feltkamp et al. (1994), Bergantinos and Vidal-Puga (2007)) and the cyclecomplete solution (Trudeau (2012))) are designed to be in the core. Given that in our context the core can be empty, there is limited appeal in extending these methods to our context. We therefore focus on the Kar solution (Kar (2002)), which is the Shapley value of the stand-alone game.

Kar (2002) characterizes his solution with Group Independence, which implies that if there are never any gains for two groups to cooperate on the construction of the network, then we can compute the cost shares independently for each group. It also uses the property of Equal Treatment, which implies that if the cost of edge $(i, j)$ goes down, the cost shares of agents $i$ and $j$ should change by the same amount. This notion is very natural as the edge that generates the saving is jointly owned by the two agents. If Group Independence can be extended easily to our context, it is not trivial to adapt Equal Treatment. It seems natural to keep the requirement if $i$ and $j$ are identical with respect to their desire to connect to the source, as that edge can be used to connect $i$ or $j$ to the source. If $i$ wants to be connected but not $j$, it does not seem as natural to require them to have equal changes in their cost shares, as the agents play different roles. In particular, the edge can only be used to connect $i$. Therefore, we only require that the changes be proportional to each other. This is a consistency requirement, as this guarantees that the way we treat demanders versus non-demanders remains constant throughout. In particular, that treatment does not depend on the magnitude of the change in cost, the way it affects the optimal configuration or the identity of the agents involved.

When we adapt Equal Treatment to cases in which agents have different desire to connect to the source, if we require that a demander and a non-demander be both (strictly) affected by the change in cost of the edge between them, we characterize a family of weighted Shapley values. We then describe three interesting weighted Shapley values. The first one is obtained by requiring that when the cost of an edge between a demander and a non-demander decreases, both agents are affected in the same manner. The other two methods considered are limits of the characterized family of weighted Shapley values when the impact on one type of agents tends to zero.

We then introduce a series of properties to differentiate the three solutions. These properties are related to stability, the rent given to non-demanders and the non-manipulability of demands. The properties are used to characterize the three weighted Shapley values.

The paper is divided as follows: Section 2 describes the model and provides an example of how the optimal configuration is computed. Section 3 shows that the core can be empty. Section 4 describes and characterizes the family of methods obtained by extending Kar's characterization for mest problems. Section 5 differentiates and characterizes three of those methods. As our model covers many different cases explored in the literature, links with these models are clarified in Section 6.

\section{The model}

In order to define mcst problems with indifferent agents, we first define classic mcst problems.

\footnotetext{
${ }^{2}$ Negative externalities coming with the network are another reason for conflict. This aspect of the problem is not modelled here.
} 


\subsection{Minimum cost spanning tree problems}

Let $\mathcal{N}=\{1,2, \ldots\}$ be the set of potential participants and $N \subseteq \mathcal{N}$ be the set of actual participants that need to be connected to the source, denoted by 0 . Let $N_{0}=N \cup\{0\}$. For any set $Z \subseteq \mathcal{N} \cup\{0\}$, define $Z^{p}$ as the set of all non-ordered pairs $(i, j)$ of elements of $Z$. In our context, any element $(i, j)$ of $Z^{p}$ represents the edge between $i$ and $j$. We often write a generic element of $Z^{p}$, an edge, as $e$. Let $c=\left(c_{e}\right)_{e \in N_{0}^{p}}$ be a vector in $\mathbb{R}_{+}^{N_{0}^{p}}$ with $c_{e}$ representing the cost of edge $e$. Let $\Gamma(N)$ be the set of all cost vectors when the set of agents is $N$, with $N \subseteq \mathcal{N}$. Let $\Gamma$ be the set of all cost vectors, for all possible $N$. Since $c$ assigns a cost to all edges $e$, we often abuse language and call $c$ a cost matrix. A minimum cost spanning tree problem is a triple $(0, N, c)$. Since 0 does not change, we omit it in the following and simply identify a mcst problem as $(N, c)$, with $N \subseteq \mathcal{N}$ and $c \in \Gamma(N)$.

A cycle $p_{l l}$ is a set of $K \geq 3$ edges $\left(i_{k}, i_{k+1}\right)$, with $k \in[0, K-1]$ and such that $i_{0}=i_{K}=l$ and $i_{1}, \ldots, i_{K-1}$ distinct and different than $l$. A path $p_{l m}$ between $l$ and $m$ is a set of $K$ edges $\left(i_{k}, i_{k+1}\right)$, with $k \in[0, K-1]$, containing no cycle and such that $i_{0}=l, i_{K}=m$ and $i_{1}, \ldots, i_{K-1}$ distinct and different than $l$ and $m$. A spanning tree is a non-orientated graph without cycles that connects all elements of $N_{0}$. A spanning tree $t$ is identified by the set of its edges. Its associated cost is $\sum_{e \in t} c_{e}$.

In a classic mcst problem, the minimum cost of connecting $N$ to the source and the associated minimum cost spanning tree is obtained using Prim's algorithm, which has $|N|$ steps. First, pick an edge $(0, i)$ such that $c_{0 i} \leq c_{0 j}$ for all $j \in N$. We then say that $i$ is connected. In the second step, we choose an edge with the smallest cost connecting an agent in $N \backslash\{i\}$ either directly to the source or to $i$, which is connected. We continue until all agents are connected, at each step connecting an agent not already connected to an agent already connected or to the source. Let $\hat{C}(N, c)$ be the associated cost. Let $c^{S}$ be the restriction of the cost matrix $c$ to the coalition $S_{0} \subseteq N_{0}$. Let $\hat{C}(S, c)$ be the cost of the mcst problem $\left(S, c^{S}\right)$.

For a mcst problem $(N, c)$, a cost allocation $y \in \mathbb{R}^{N}$ assigns a cost share to each agent, and the budget balance condition is $\sum_{i \in N} y_{i}=\hat{C}(N, c)$.

A cost sharing solution (or rule) assigns a cost allocation $y(N, c)$ to any admissible mcst problem $(N, c)$. The Kar solution (Kar (2002)) is the Shapley value of the stand-alone cost game $\hat{C}: y^{K}(N, c)=$ $\operatorname{Sh}(\hat{C}(\cdot, c))$.

\subsection{Minimum cost spanning tree problems with indifferent agents}

To define minimum cost spanning tree problems with indifferent agents, we need to distinguish agents that need a connection to the source from those that do not. Let $N^{d} \subseteq \mathcal{N}$ be the set of players that desire a connection to the source. Let $N^{t} \subseteq \mathcal{N} \backslash N^{d}$ be the set of players that are not interested in being connected to the source but are ready to let others use their locations to connect to the source. Again, since 0 does not change, we omit it in the following and simply identify a mcst problem with indifferent agents as $\left(\left(N^{d}, N^{t}\right), c\right)$, with $N^{d} \cup N^{t}=N \subseteq \mathcal{N}$ and $c \in \Gamma(N)$.

To obtain the cost for a mcst problem with indifferent agents $\left(\left(N^{d}, N^{t}\right), c\right)$, we proceed as follow. First, we compute the cost function $\hat{C}$ of the mest problem as if all agents wanted to be connected to the source. Then, for a coalition $S \subseteq N$, the cost $C\left(S,\left(\left(N^{d}, N^{t}\right), c\right)\right.$ is the cost to connect agents in $S \cap N^{d}$, with the help of agents in $S \cap N^{t}$. Therefore, $C\left(S,\left(\left(N^{d}, N^{t}\right), c\right)=\min _{T \subseteq\left(N^{t} \cap S\right)} \hat{C}\left(S \cap N^{d} \cup T, c\right)\right.$ with the convention that $\hat{C}(\emptyset, c)=0$. Given these definitions, we say that $C$ is the stand-alone cost function associated with the problem $\left(\left(N^{d}, N^{t}\right), c\right)$.

Figure 1 shows an example with 3 players, with identity of the players appearing in bold. Other numbers are the cost of the corresponding edges. Here are the corresponding values for $\hat{C}$ and $C$, first when both agents 2 and 3 want to be connected, then when only agent 3 has such a demand. 


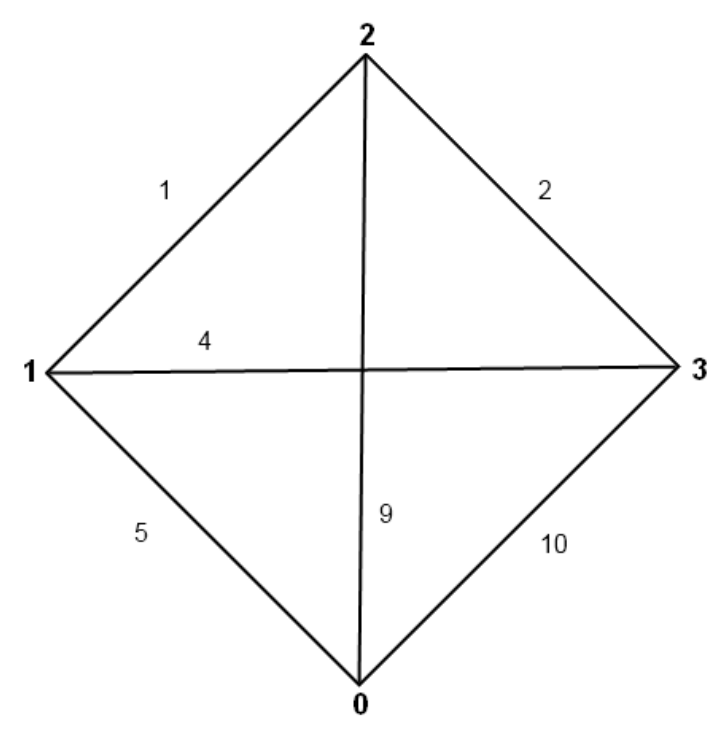

Figure 1: Example with 3 players

$\begin{array}{cccc}S & \hat{C}(S) & C(S,((\{2,3\},\{1\}), c) & C(S,((\{3\},\{1,2\}), c) \\ \{1\} & 5 & 0 & 0 \\ \{2\} & 9 & 9 & 0 \\ \{3\} & 10 & 10 & 10 \\ \{1,2\} & 6 & 6 & 0 \\ \{1,3\} & 9 & 9 & 9 \\ \{2,3\} & 11 & 11 & 10 \\ \{1,2,3\} & 8 & 8 & 8\end{array}$

For example, to compute $C(\{1,3\},((\{2,3\},\{1\}), c)$, either agent 3 connects without help at a cost of $\hat{C}(\{3)\}=10$ or with the help of agent 1 at a cost of $\hat{C}(\{1,3)\}=9$.

A cost sharing solution assigns a cost allocation $y\left(\left(N^{d}, N^{t}\right), c\right)$ to any admissible mcst problem with indifferent agents $\left(\left(N^{d}, N^{t}\right), c\right)$.

\section{Possible emptiness of the core}

As in the Steiner tree problem, and unlike in minimum cost spanning tree problems, the core can be empty for mcst problems with indifferent agents. This means that we might not be able to find an allocation that removes for all coalitions the incentives to secede from the large group. Formally, the core is the set of allocations $y \in \mathbb{R}^{N}$ such that $\sum_{i \in S} y_{i} \leq C\left(S,\left(N^{d}, N^{t}\right), c\right)$ for all $S \subset N$ and $\sum_{i \in N} y_{i}=C\left(N,\left(N^{d}, N^{t}\right), c\right)$. The following example shows that it can be empty. ${ }^{3}$

We have an illustration of cost matrix $c$ in Figure 2, with represented edges having a cost of one while unrepresented edges have a cost of ten. Suppose that $N^{d}=\{1,2,3\}$ and $N^{t}=\{4,5,6\}$. It is easy to see that the cost for the grand coalition is 5 , while the costs for coalitions $\{1,2,5\},\{1,3,4\}$

\footnotetext{
${ }^{3}$ This example was provided by Gustavo Bergantinos, Juan Vidal-Puga, Maria Gomez-Rua and Leticia Lorenzo during a visit at the University of Vigo.
} 


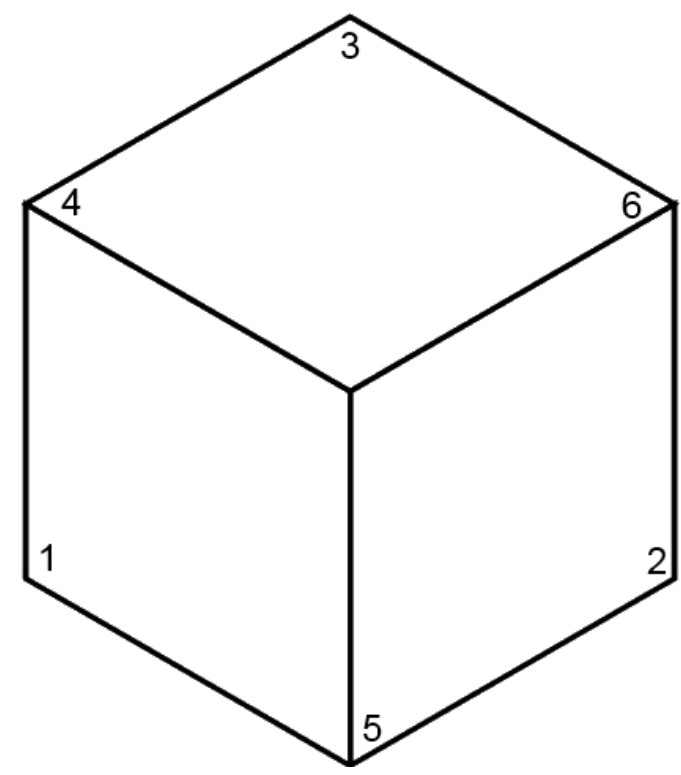

Figure 2: Example with empty core

and $\{2,3,6\}$ are all of 3 . This generates the following core constraints:

$$
\begin{aligned}
& y_{1}+y_{2}+y_{5} \leq 3 \\
& y_{1}+y_{3}+y_{4} \leq 3 \\
& y_{2}+y_{3}+y_{6} \leq 3 .
\end{aligned}
$$

Summing these up, we obtain

$$
\begin{aligned}
2\left(y_{1}+y_{2}+y_{3}\right)+y_{4}+y_{5}+y_{6} & \leq 9 \\
y_{1}+y_{2}+y_{3} & \leq 4\left(\text { using } \sum_{i=1}^{6} y_{i}=5\right)
\end{aligned}
$$

This means that $y_{4}+y_{5}+y_{6} \geq 1$. However, since $N^{t}=\{4,5,6\}, C\left(\{4,5,6\},\left(N^{d}, N^{t}\right), c\right)=0$. Thus, the core is empty.

\section{Extending Kar's characterization}

Kar's characterization uses two properties: Group Independence and Equal Treatment. ${ }^{4}$ Group Independence conveys the idea that if two groups of agents always connect to the source in an independent manner, then computing the cost shares on the whole group or independently on the two groups makes no difference. This is a natural property, without which one group might subsidize the other for costs for which it is clearly not responsible. The concept applies as well to our problem as it does to mcst problems. To formally define the property we define irrelevant edges as follows: for $i, j \in N$, we say that $(i, j)$ is an irrelevant edge if $c_{i j} \geq c_{0 i}, c_{0 j}$. Otherwise, we say it is a relevant edge. Relevant edges will be used by coalition $\{i, j\}$ if both agents want to connect to the source and nobody cooperates with them. Irrelevant edges will not be used in that situation, or in any other.

\footnotetext{
${ }^{4}$ To be exact, Group Independence is divided in two distinct properties in Kar (2002). However, a solution satisfies these two properties if and only if it satisfies Group Independence.
} 
Group Independence: if there exists $S \subseteq N^{d}$ and $T \subseteq N^{t}$ such that $(i, j)$ is an irrelevant edge for all $i \in S \cup T, j \in N \backslash(S \cup T)$, then $y_{i}\left(\left(N^{d}, N^{t}\right), c\right)=y_{i}\left((S, T), c^{S \cup T}\right)$ if $i \in S \cup T$ and $y_{i}\left(\left(N^{d}, N^{t}\right), c\right)=y_{i}\left(\left(N^{d} \backslash S, N^{t} \backslash T\right), c^{N \backslash(S \cup T)}\right)$ if $i \in N \backslash(S \cup T)$.

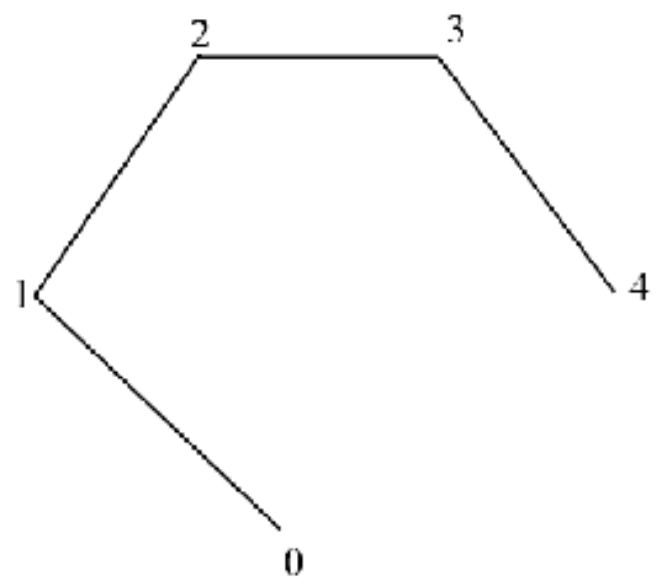

Figure 3: Illustration of Equal treatment

Consider the cost matrix illustrated in Figure 3 in which the represented edges have a cost of 2, with the others having a cost of 10 . Suppose that all agents want to be connected to the source. The mcst is exactly the set of represented edges and has a total cost of 8. Suppose that the cost of one of the edges in $\{(1,2),(2,3),(3,4)\}$ decreases to 1 . This generates a saving of 1 . How should we allocate this saving? In particular, how should the two agents at the extremities of this edge be affected? Kar (2002) uses the Equal Treatment principle: since the agents have an equal property right to an edge between them, they should be affected equally so both agents receive the same reduction in their cost share. Notice that this principle does not mean that each agent gains half of the savings: the shares of other agents could also be affected, with the combined changes to the shares of the agents at the extremities possibly larger or smaller than the savings. Formally, we adapt the Equal Treatment principle to our setting as follows:

Equal Treatment: Let $c, c^{\prime}$ be such that $c_{i j}^{\prime}<c_{i j}$ for $i, j \in N$ and $c_{e}^{\prime}=c_{e}$ for $e \neq(i, j)$. Then,

$$
y_{i}\left(\left(N^{d}, N^{t}\right), c^{\prime}\right)-y_{i}\left(\left(N^{d}, N^{t}\right), c\right)=y_{j}\left(\left(N^{d}, N^{t}\right), c^{\prime}\right)-y_{j}\left(\left(N^{d}, N^{t}\right), c\right) .
$$

What makes this property particularly attractive for mcst problems is the fact that in addition to a pair of agents having equal property rights to the edge that runs between them, they also have an equal desire to be connected to the source, which further justifies that they should be affected in the same manner.

Consider again the cost matrix illustrated in Figure 3, but now suppose that $N^{d}=\{3,4\}$. If the cost of the edge $(3,4)$ decreases, it seems very natural to impose Equal Treatment. When used, the edge $(3,4)$ allows the two agents to pay for a single connection from them to the source, instead of two, generating a surplus for both agents. It also seems very natural to impose Equal Treatment if it is the cost of the edge $(1,2)$ that decreases. Since these two agents are indifferent to a connection to the source, this edge can only be used in a joint bid to offer a cheaper connection to agents that want to be connected to the source. We formalize this idea as follows:

Equal Treatment for Equal Demanders (ETED): Let $c, c^{\prime}$ be such that for $i, j \in N, c_{i j}^{\prime}<c_{i j}$ and $c_{e}^{\prime}=c_{e}$ for $e \neq(i, j)$. If $i, j \in N^{d}$ or $i, j \in N^{t}$, then

$$
y_{i}\left(\left(N^{d}, N^{t}\right), c^{\prime}\right)-y_{i}\left(\left(N^{d}, N^{t}\right), c\right)=y_{j}\left(\left(N^{d}, N^{t}\right), c^{\prime}\right)-y_{j}\left(\left(N^{d}, N^{t}\right), c\right) .
$$


By contrast, suppose that it is the cost of edge $(2,3)$ in Figure 3 that decreases to 1 . Should the cost shares of agents 2 and 3 change in the same manner? This is not as clear. One could argue that the demander should receive a greater discount, as without the demand of agent 3 , the edge $(2,3)$ is not valuable. Alternatively, the argument can be made that the non-demander should receive a greater discount: in particular, if we believe that such an agent, who has no desire to be connected to the source, might back out of the project, or if we believe that the good or service is undesirable (for example if the good is a pollutant). We are thus trying to weight the value of the desire of the demanders to be connected to the source against the value of the locations of the non-demanders. Equal treatment imposes that we weight these equally, something we might not want to do. However, once we have determined how the change of the demander compares to the change of the non-demander, it seems appropriate to proceed in the same manner whenever we face a similar situation in which we need to divide a surplus between a demander and a non-demander. Suppose that for every unit of savings that the demander obtains, the non-demander gets $\beta$. Holding this ratio constant is a consistency property: the weight we put on demanders versus non-demanders, whatever it is, remains constant in all circumstances. This leads to the following property:

Consistent Treatment for Unequal Demanders (CTUD): Let $c, c^{\prime}$ such that for $i \in N^{d}$ and $j \in N^{t}, c_{i j}^{\prime}<c_{i j}$ and $c_{e}^{\prime}=c_{e}$ for $e \neq(i, j)$. For all such pairs of matrices $c, c^{\prime}$, we have

$$
\alpha\left[y_{i}\left(\left(N^{d}, N^{t}\right), c^{\prime}\right)-y_{i}\left(\left(N^{d}, N^{t}\right), c\right)\right]=(1-\alpha)\left[y_{j}\left(\left(N^{d}, N^{t}\right), c^{\prime}\right)-y_{j}\left(\left(N^{d}, N^{t}\right), c\right)\right]
$$

with $\alpha \in[0,1]$.

With $\beta \equiv \frac{1-\alpha}{\alpha}$, we can write the above equality as

$$
\left[y_{i}\left(\left(N^{d}, N^{t}\right), c^{\prime}\right)-y_{i}\left(\left(N^{d}, N^{t}\right), c\right)\right]=\beta\left[y_{j}\left(\left(N^{d}, N^{t}\right), c^{\prime}\right)-y_{j}\left(\left(N^{d}, N^{t}\right), c\right)\right]
$$

which fits with the description given above. However, our choice of notation allows us to define three interesting cases. If $\alpha=0$, then $j \in N^{t}$ is unaffected by the change. If $\alpha=1$, then the cost share of $i \in N^{d}$ stays constant. Finally, if $\alpha=\frac{1}{2}$, it is just as if we applied Equal Treatment to agents that do not have the same demand.

We restrict the possible values of $\alpha$ to $[0,1]$. In addition to being difficult to interpret, any value of $\alpha$ outside of the interval would lead the share of one agent to increase and the other to decrease. Suppose that we start with a case in which all edges are irrelevant, meaning that by Group Independence all agents pay their stand-alone cost. If an edge between a demander and a non-demander becomes cheaper, by CTUD with $\alpha \notin[0,1]$, one of the two agents would end up with a share larger than its stand-alone cost.

The solutions satisfying Group Independence, Equal Treatment for Equal Demanders and Consistent Treatment for Unequal Demanders are weighted Shapley values. We adapt the definition of Kalai and Samet (1987) to our context.

Let $\Pi(N)$ be the set of permutations of $N$. Let $B(i, \pi)$ represent the set of agents coming in before $i$ in $\pi$. We have, for $\theta \in(0,1)$ and $i \in N$, that

$$
W S_{i}^{\theta}\left(\left(N^{d}, N^{t}\right), c\right)=\sum_{\pi \in \Pi(N)} p(\pi)\left[C\left(B(i, \pi) \cup\{i\},\left(N^{d}, N^{t}\right), c\right)-C\left(B(i, \pi),\left(N^{d}, N^{t}\right), c\right)\right]
$$

where

$$
\begin{aligned}
p(\pi) & =\prod_{k=0}^{n-1} \frac{w_{\pi_{n-k}}}{\sum_{l=1}^{n-k} w_{\pi_{l}}} \text { is the weight of permutation } \pi, \text { with } \\
w_{i} & =\left\{\begin{array}{c}
\theta \text { if } i \in N^{t} \\
1-\theta \text { if } i \in N^{d}
\end{array}\right.
\end{aligned}
$$

The solution $W S^{\theta}$ is the weighted Shapley value of game $C\left(\cdot,\left(N^{d}, N^{t}\right), c\right)$ in which each agent $i \in N^{d}$ has a weight of $1-\theta$ and each agent $i \in N^{t}$ has a weight of $\theta$. Notice that the weights help us 
determine who is added to the group last. If we have only two agents, a demander and a non-demander, the demander is added last with probability $1-\theta$. As an illustration if $N=\{1,2,3\}$, to obtain the permutation $\pi=(3,2,1)$, we need to pick agent 1 first, which happens with probability $\frac{w_{1}}{w_{1}+w_{2}+w_{3}}$. We also need to pick agent 2 second, which then occurs with probability $\frac{w_{2}}{w_{2}+w_{3}}$. The weight (probability) of permutation $(3,2,1)$ is thus $\frac{w_{1}}{w_{1}+w_{2}+w_{3}} \frac{w_{2}}{w_{2}+w_{3}}$.

We show that with Group Independence, the adaptations of Equal Treatment imply a unique solution (for each value of the parameter in CTUD). Since ETED, CTUD and budget balance give us a system of equations relating the cost shares to each other, the strategy of the proof is to write this system in matrix form and to show that it has a unique solution, with this solution being a weighted Shapley value. To prove the main result, we first need the following technical result on a family of matrices that appear in the proof.

Lemma 1 Let $\Psi$ be the set of square matrices such that if $M \in \Psi$ is of dimension $m \times m$, we have

$$
\begin{aligned}
M_{l l} & >0 \text { for } l=1, \ldots, m \\
M_{k l} & \leq 0 \text { for } l>k \\
M_{k l} & =0 \text { for } l<k<m \\
M_{m l} & =1 \text { for } l=1, \ldots, m
\end{aligned}
$$

Then, for any $M \in \Psi, \operatorname{det}(M)>0$.

We are now ready for the main result.

Theorem 1 For a given value $\alpha \in(0,1)$, with $\alpha$ the parameter in Consistent Treatment for Unequal Demanders, $W S^{\alpha}$ is the unique cost sharing solution satisfying Group Independence, Equal Treatment for Equal Demanders and Consistent Treatment for Unequal Demanders.

Proof. Lemma 3 in Appendix shows that $W S^{\alpha}$ satisfies all properties. We show that the properties imply a unique solution.

First, suppose that $c$ has no relevant edges. By multiple applications of Group Independence and budget balance, we have $y_{i}\left(\left(N^{d}, N^{t}\right), c\right)=y_{i}\left((\{i\}, \emptyset), c^{\{i\}}\right)=c_{0 i}$ if $i \in N^{d}$ and $y_{i}\left(\left(N^{d}, N^{t}\right), c\right)=$ $y_{i}\left((\emptyset,\{i\}), c^{\{i\}}\right)=0$ if $i \in N^{t}$.

By Group Independence, we can suppose that for any $S$ in $N$, there exists a relevant edge $(i, j)$, with $i \in S$ and $j \in N \backslash S$. (If this was not the case we could apply Group Independence and $y_{i}\left(\left(N^{d}, N^{t}\right), c\right)=$ $y_{i}\left(\left(N^{d} \cap S, N^{t} \cap S\right), c^{S}\right)$ for all $i \in S$. $)$.

The above assumption means that there is a tree $t$ in $N$ such that all edges on the tree are relevant.

Suppose that for $k, l \in N,(k, l)$ is a relevant edge. Let $c^{k l}$ be such that $c_{k l}^{k l}=\max \left\{c_{0 k}, c_{0 l}\right\}$ and $c_{e}^{k l}=c_{e}$ for all $e \neq(k, l)$. In the following, $\left(N^{d}, N^{t}\right)$ is fixed. To simplify the notation, we write $y(c)$ for $y\left(\left(N^{d}, N^{t}\right), c\right)$ where there is no confusion.

The strategy of the proof is as follows. Suppose we have $n$ agents and a tree of relevant edges linking all agents. One by one, we modify the cost of each edge to make it irrelevant. ETED or CTUD give us an equation linking the changes in the shares of the agents at the extremities of the affected edge. Combined with the budget balance, this gives us $n$ equations. We show that the system of equations always has a unique solution.

i) we have $n$ agents and $n-1$ relevant edges

This case is such that there is a single tree with relevant edges that links all agents together. We show that the system of equations has a unique solution by using a recursive argument on the number of agents. Suppose that we have a unique solution whenever we have $m-1$ or less agents and there is a single tree of relevant edges linking these agents.

We build the system of equations algorithmically as follows:

Step 1: take an agent $i$ such that $i$ belongs to only one edge in $t$ and relabel him agent 1 . By the definition of a tree, there exists such agent. Let $q(1) \in N$ be the agent such that $(1, q(1)) \in t$. 
By ETED or CTUD, there exists an equation linking $y_{1}(c)-y_{1}\left(c^{1, q(1)}\right)$ and $y_{q(1)}(c)-y_{q(1)}\left(c^{1, q(1)}\right)$. We have that in $c^{1, q(1)}$, agent 1 has no relevant edge to anybody else, and for the remaining agents, there is a single tree of relevant edges linking all of them. By Group Independence and the recursive argument, the values $y\left(c^{1, q(1)}\right)$ are well defined. Rewrite the equation as $A_{1} y_{1}(c)+B_{1} y_{q(1)}(c)=X_{1}$.

Step $k$. Let $t^{k}$ be the tree $t$ to which we have removed the agents labeled as 1 through $k-1$ in the corresponding steps. Notice that since at each step we are removing a leaf (for the current tree), $t^{k}$ is also a tree.

Take an agent $i$ such that $i$ belongs to only one edge in $t^{k}$ and relabel him agent $k$. By the definition of a tree, there exists such an agent. Let $q(k)$ be the agent such that $(k, q(k)) \in t^{k}$. By ETED or CTUD, there exists an equation linking $y_{k}(c)-y_{k}\left(c^{k, q(k)}\right)$ and $y_{q(k)}(c)-y_{q(k)}\left(c^{k, q(k)}\right)$. By Group Independence and the recursive argument the values $y\left(c^{k, q(k)}\right)$ are well defined. Rewrite the equation as $A_{k} y_{k}(c)+B_{k} y_{q(k)}(c)=X_{k}$.

Conclude at step $n-1$.

Notice that by definition, $q(k)>k$ for all $k=1, \ldots, n-1$. Build a matrix $Z$ of dimension $n \times n$ in the following manner. At line $k$, insert $A_{k}$ in position $k, B_{k}$ in position $q(k)$ and 0 otherwise, for $k=1, \ldots, n-1$. The $n^{\text {th }}$ equation is the budget balance condition: insert 1 at position $(n, i)$ for each $i=1, \ldots, n$. Let $X_{N}=C\left(N,\left(N^{d}, N^{t}\right), c\right)$ and $X$ the column vector of $\left(X_{1}, \ldots, X_{N}\right)$. We have that $Z y=X$. To solve for $y$, we need to inverse $Z$.

Notice first that the possible values for $A_{k}$ are $\{1, \alpha, 1-\alpha\}^{5}$ and are all strictly positive by the assumption that $\alpha \in(0,1)$. The possible values for $B_{k}$ are $\{-1,-\alpha, \alpha-1\}$ and are all strictly negative since $\alpha \in(0,1)$. Therefore, $Z_{l l}=A_{l}>0$ and $Z_{k l}=B_{l}<0$ if $k=q(l)$ and $Z_{k l}=0$ otherwise, for $l<n$. Thus, $Z \in \Psi$. By Lemma $1, \operatorname{det}(Z)>0$ and, therefore, the system of equations in $Z y=X$ has a unique solution. By the recursive argument, since it is true for one agent, we have a unique solution for any number of agents $n$ if we have $n-1$ relevant edges connecting them.

ii) we have $n$ agents and $m \geq n$ relevant edges

This case is such that we have many trees made of relevant edges that connect all agents. We show that the system of equations has a unique solution by proceeding recursively on the number of relevant edges. Suppose that we have a unique solution whenever we have less than $m$ relevant edges. Notice that for $n-1$ relevant edges, the cost sharing values were found in i).

Build the matrices $Z$ and $X$ as in step i). At any step $k$ of the algorithm, $c^{k, q(k)}$ contains $m-1$ relevant edges. If there it does not contain a tree in $N$ such that all edges on the tree are relevant, we can apply Group Independence and step i) to find the values $y\left(c^{k, q(k)}\right)$. If $c^{k, q(k)}$ containsa tree in $N$ such that all edges on tree are relevant, by i) and the recursive argument the values $y\left(c^{k, q(k)}\right)$ are well defined.

As in i), $Z \in \Psi$ and the system of equations in $Z y=X$ has a unique solution.

From i) and ii) we can conclude that we have a unique solution for any number of agents and any number of relevant edges, thus covering all possible cases.

Lemma 4 in Appendix shows that the properties are independent.

Remember that while defining the CTUD property, we identified three natural values for $\alpha: 0, \frac{1}{2}$ and 1. When $\alpha=\frac{1}{2}$, we have a weighted Shapley value in which demanders and non-demanders have the same weight. Thus, we are back with a standard Shapley value. The resulting solution is nothing more than a straightforward extension of the Kar solution, i.e. the Shapley value of $\left.C\left(\cdot,\left(N^{d}, N^{t}\right), c\right)\right)$. Formally, let

$$
\begin{aligned}
y^{K}\left(\left(N^{d}, N^{t}\right), c\right) & =W S^{\frac{1}{2}}\left(C\left(\cdot,\left(N^{d}, N^{t}\right), c\right)\right) \\
& =\operatorname{Sh}\left(C\left(\cdot,\left(N^{d}, N^{t}\right), c\right)\right) .
\end{aligned}
$$

Unfortunately, Theorem 1 does not cover the cases in which $\alpha \in\{0,1\}$. In fact, for these values, CTUD tells us that when the cost of an edge drops, one agent at its extremities is unaffected, but we

\footnotetext{
${ }^{5}$ If we use ETED, we have that $A_{k}=B_{k}=1$. If we use CTUD and $k \in N^{d}$, we have that $A_{k}=\alpha$ and $B_{k}=\alpha-1$. If we use CTUD and $k \in N^{t}$, we have that $A_{k}=1-\alpha$ and $B_{k}=-\alpha$.
} 
learn nothing about the effect on the other agent. We thus lose uniqueness since many methods can satisfy CTUD for these values of the parameter.

A closer look at the definition of weighted Shapley values helps us solve this problem. In the definition of $p(\pi)$, the weight of permutation $\pi$, we have that the probability of picking agent $\pi_{n-k}$ as the $k^{t h}$-before-last agent in the permutation is $\frac{w_{\pi_{n-k}}}{\sum_{l=1}^{n-k} w_{\pi_{l}}}$. If $\alpha \in\{0,1\}$, we might have that $\sum_{l=1}^{n-k} w_{\pi_{l}}=0$, in which case the probability is not well defined. If $\alpha=0$, that case would occur if all remaining agents belong to $N^{t}$. For $\alpha=1$, it is when all agents belong to $N^{d}$. In both cases, since agents in the same group have the same weight, it would be natural to define that probability as $\frac{1}{m}$, where $m$ is the number of remaining agents. We adjust our definition for this. We have, for $\theta \in[0,1]$, that

$$
\overline{W S}_{i}^{\theta}\left(\left(N^{d}, N^{t}\right), c\right)=\sum_{\pi \in \Pi(N)} p(\pi)\left[C\left(B(i, \pi) \cup\{i\},\left(N^{d}, N^{t}\right), c\right)-C\left(B(i, \pi),\left(N^{d}, N^{t}\right), c\right)\right]
$$

with

$$
\begin{aligned}
& p(\pi)=\left\{\begin{array}{c}
\prod_{k=0}^{\bar{k}-1} \frac{w_{\pi_{n-k}}^{\prime}}{\sum_{l=1}^{n-k} w_{\pi_{l}}^{\prime}} \prod_{l=1}^{n-\bar{k}} \frac{1}{l} \text { if } \sum_{l=1}^{n-m} w_{\pi_{l}}^{\prime}=0 \text { for all } n-1 \geq m \geq \bar{k} \\
\prod_{k=0}^{n-1} \frac{w_{\pi_{n-k}}^{\prime}}{\sum_{l=1}^{n-k} w_{\pi_{l}}^{\prime}} \text { otherwise }
\end{array}\right. \\
& w_{i}=\left\{\begin{array}{c}
\theta \text { if } i \in N^{t} \\
1-\theta \text { if } i \in N^{d}
\end{array} .\right.
\end{aligned}
$$

We define $y^{0}$ as a variant of the Shapley-Shubik method that disregards the contributions of agents in $N^{t}$. Let $\bar{C}$ be defined for all $S \subseteq N^{d}$ and such that $\bar{C}\left(S,\left(N^{d}, N^{t}\right), c\right)=C\left(S \cup N^{t},\left(N^{d}, N^{t}\right), c\right)$. Then, $y_{i}^{0}\left(\left(N^{d}, N^{t}\right), c\right)=S h_{i}\left(\bar{C}\left(\cdot,\left(N^{d}, N^{t}\right), c\right)\right)$ for all $i \in N^{d}$ and $y_{i}^{0}\left(\left(N^{d}, N^{t}\right), c\right)=0$ for all $i \in N^{t}$. Notice that to compute $\bar{C}$, we assume that demanders can always use the locations of non-demanders as they wish, without providing compensations. We can verify that $y^{0}=\overline{W S}^{0}$.

Finally, we define $y^{1}$ as a solution that takes the opposite stand and assigns all of the gains coming from technological cooperation to the agents in $N^{t}$. Let $C^{N^{d}}$ be defined for all $S \subseteq N^{d}$ and such that $C^{N^{d}}\left(S,\left(N^{d}, N^{t}\right), c\right)=C\left(S,\left(N^{d}, N^{t}\right), c\right)$. Let $\tilde{C}$ be defined for all $S \subseteq N^{t}$ and such that $\tilde{C}\left(S,\left(N^{d}, N^{t}\right), c\right)=C\left(S \cup N^{d},\left(N^{d}, N^{t}\right), c\right)-C\left(N^{d},\left(N^{d}, N^{t}\right), c\right)$. Then, $y_{i}^{1}\left(\left(N^{d}, N^{t}\right), c\right)=S h_{i}\left(C^{N^{d}}\left(\cdot,\left(N^{d}, N^{t}\right), c\right)\right)$ for all $i \in N^{d}$ and $y_{i}^{1}\left(\left(N^{d}, N^{t}\right), c\right)=S h_{i}\left(\tilde{C}\left(\cdot,\left(N^{d}, N^{t}\right), c\right)\right)$ for all $i \in N^{t}$. Here, $C^{N^{d}}$ is as if demanders never had access to the locations of non-demanders. $\tilde{C}$ is the function assigning the cost savings generated by the locations of any coalition of non-demanders. We can verify that $y^{1}=\overline{W S}^{1}$.

It is also easy to verify that $\overline{W S}^{0}$ and $\overline{W S}^{1}$ are the limits of $W S^{\alpha}$ when $\alpha$ tends to zero and one, respectively.

We verify in Lemma 5 that $y^{0}$ and $y^{1}$ satisfy Group Independence, ETED and CTUD for, respectively, $\alpha=0$ and $\alpha=1$.

Example 1 We provide an example to illustrate the solutions proposed above. We find in Figure 4 the structure, with agents appearing in bold. Other numbers are the cost of the corresponding edges.

\{Insert Figure 4: Illustration of cost sharing\}

The following table provides the cost shares prescribed by $y^{0}, y^{K}$ and $y^{1}$, as well as for the case with a generic $\alpha$. Shares are computed for two cases, first when only agent 3 wants to be connected, then when agents 2 and 3 need to be connected.

$$
\begin{array}{ccc} 
& N^{d}=\{3\} & N^{d}=\{2,3\} \\
y^{0} & (0,0,4) & \left(0, \frac{3}{2}, \frac{5}{2}\right) \\
y^{K} & \left(-\frac{11}{18},-\frac{11}{18}, \frac{47}{9}\right) & \left(-\frac{5}{6}, \frac{5}{3}, \frac{19}{6}\right) \\
y^{1} & \left(-\frac{3}{2},-\frac{3}{2}, 7\right) & \left(-1, \frac{3}{2}, \frac{7}{2}\right) \\
W S^{\alpha} & \left(-\frac{\alpha\left[3 \alpha^{2}+2 \alpha+1\right]}{(1+\alpha)^{2}},-\frac{\alpha\left[3 \alpha^{2}+2 \alpha+1\right]}{(1+\alpha)^{2}}, \frac{2\left[3 \alpha^{3}+4 \alpha^{2}+5 \alpha+2\right]}{(1+\alpha)^{2}}\right) & \left(\frac{3 \alpha^{2}-4 \alpha}{2-\alpha}, \frac{6-\alpha-2 \alpha^{2}}{2(2-\alpha)}, \frac{10+\alpha-4 \alpha^{2}}{2(2-\alpha)}\right)
\end{array}
$$




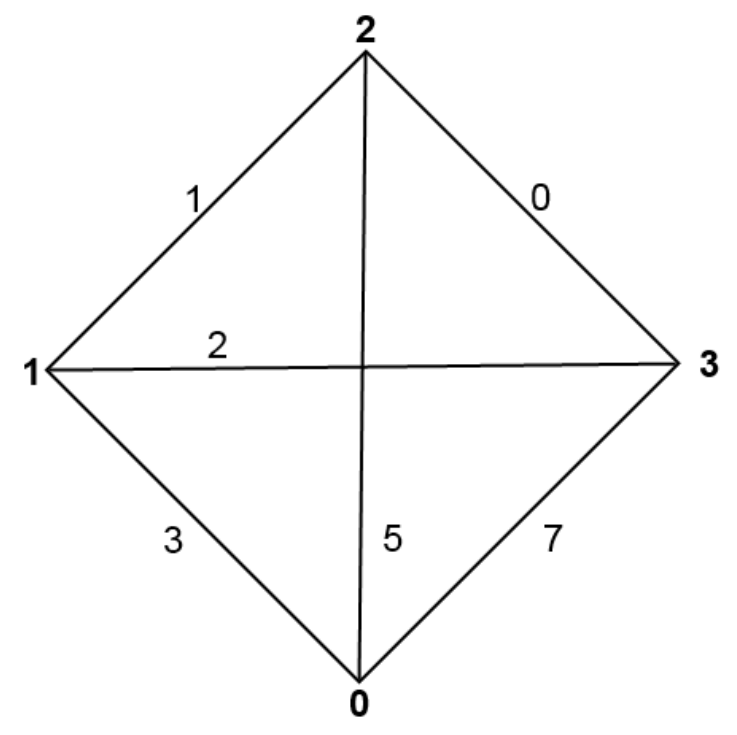

Figure 4: Illustration of cost sharing

A first observation is that $\alpha$ enters the cost shares in a non-linear fashion and thus $y^{K}$ is not a weighted average of $y^{0}$ and $y^{1}$. When $N^{d}=\{1,2,3\}$, we have a classic mest problem. In this case, CTUD is not necessary, as all agents have an equal desire to be connected to the source. Thus, all methods prescribe the same cost shares of $\left(\frac{1}{6}, \frac{7}{6}, \frac{8}{3}\right)$. It is also interesting to see that even though we have the intuition that a higher $\alpha$ is more favorable to agents in $N^{t}$, cost shares for these agents are not necessarily decreasing in $\alpha$. For example, $y_{1}((\{2,3\},\{1\}), c)$ reaches a minimum at $\alpha=\frac{6-2 \sqrt{3}}{3} \approx 0.845$. Figure 5 illustrates the weights on the various permutations, as a factor of $\alpha$. In our example, agent 1 gets nothing in the permutations in which he comes in first. The probability of such permutations decreases monotonically with $\alpha$. He gets -1 in permutations in which he comes last, and the probability of such permutations increases monotonically with $\alpha$. However, agent 1 has its most advantageous imputation $(-2)$ when $\pi=(3,1,2)$. He gets -1 for the imputation $\pi=(2,1,3)$. The weights for these permutations are zero for $\alpha \in\{0,1\}$, increase on $[0,2-\sqrt{2}]$ and decrease on $[2-\sqrt{2}, 1]$. Thus, agent 1 does not always prefer $\alpha$ to increase. Stated otherwise, in $y^{1}$, non-demanders get the credit for their contributions when we add them to the whole group of demanders, but not for their contributions to any subgroup of demanders, for which the value of the location of the non-demander might be higher.

\section{Characterizing the three solutions}

In order to distinguish the three particular methods defined in the previous section, we define a set of simple properties. We start with properties that take advantage of the differences between demanders and non-demanders. In general, when an agent wants to be connected to the source, we take into account two different factors to compute her cost share: how costly it is to connect her to the source, for which she should pay a positive share, and how much is saved by letting others connect through her location, for which she could receive a subsidy. For an agent that does not want to be connected to the source, only the second consideration matters. Following these observations, the next two properties are very natural.

Demand Monotonicity: Let $c \in \Gamma(N)$ and $i \in N^{t}$. We have that $y_{i}\left(\left(N^{d} \cup\{i\}, N^{t} \backslash\{i\}\right), c\right) \geq$ $y_{i}\left(\left(N^{d}, N^{t}\right), c\right)$.

Demand Ranking: Let $c \in \Gamma(N)$ and $i \in N^{t}, j \in N^{d}$. If $c_{i k}=c_{j k}$ for all $k \in N_{0} \backslash\{i, j\}$, then 


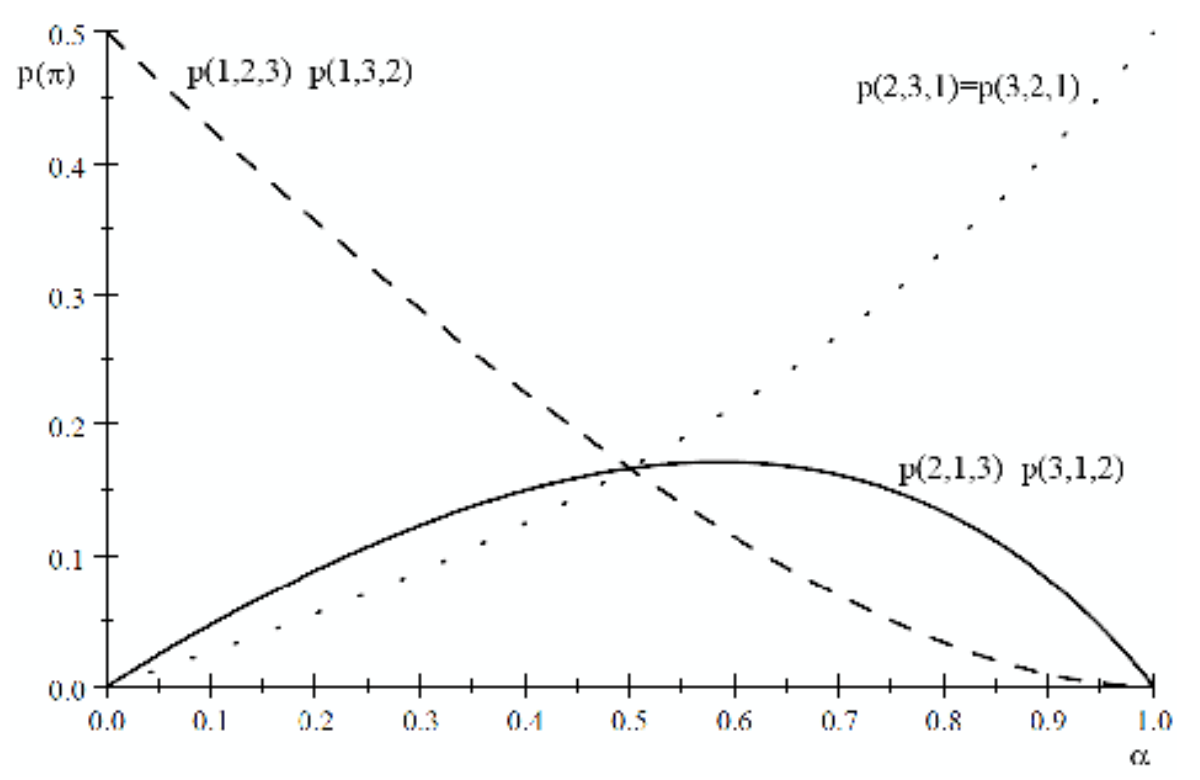

Figure 5: Weights on the various permutations

$y_{i}\left(\left(N^{d}, N^{t}\right), c\right) \leq y_{j}\left(\left(N^{d}, N^{t}\right), c\right)$.

Demand Monotonicity imposes the condition that if an agent indifferent to a connection to the source suddenly desires such a connection, his change of mind cannot decrease his cost share. In standard cost sharing models, it was introduced by Moulin (1995) and used, among many others, by Friedman and Moulin (1999) and Moulin and Sprumont (2006). Demand Ranking requires that if two agents are located in symmetric locations but only one of them wants to be connected to the source, that agent cannot pay less than the other agent. The property is similar in nature to the Fair Ranking property of Moulin and Shenker (1992). In addition to making agents responsible for their demand, Demand Monotonicity and Demand Ranking are also required if the agents can manipulate their (reported) demand to pay a smaller share. ${ }^{6}$

The final two properties deal with the rent that demanders pay to non-demanders for the use of their locations. Since we have seen that the core can be empty, we propose a weaker stability condition that requires the rent paid by demanders to non-demanders to be no larger than the savings they generate as a group. Stated otherwise, the demanders, as a group, should not be worse off when they cooperate with non-demanders. The property was introduced by Bahel and Trudeau (2013).

Fair Rent: Let $c \in \Gamma(N)$. We have that $\sum_{i \in N^{d}} y_{i}\left(\left(N^{d}, N^{t}\right), c\right) \leq C\left(N^{d},\left(N^{d}, N^{t}\right), c\right)$.

One trivial way of satisfying Fair Rent is to never subsidized any of the non-demanders. Our final property requires that solutions sometimes subsidize non-demanders. It thus eliminates solutions that completely ignore the presence of non-demanders.

Positive Rent : There exists $N^{d}, N^{t}, c \in \Gamma(N)$ and $i \in N^{t}$ such that $y_{i}\left(\left(N^{d}, N^{t}\right), c\right)<0$.

The following Lemma describes which properties are satisfied by our three solutions.

Lemma 2 i) $y^{0}$ satisfies Demand Ranking and Fair Rent but fails Demand Monotonicity and Positive Rent.

ii) $y^{K}$ satisfies Demand Monotonicity, Demand Ranking and Positive Rent but fails Fair Rent.

iii) $y^{1}$ satisfies Fair Rent and Positive Rent but fails Demand Monotonicity and Demand Ranking.

Proof. In Appendix.

The properties can also be used to characterize the solutions.

\footnotetext{
${ }^{6}$ In the mest context, manipulability in the form of merging was studied in Gomez-Rua and Vidal-Puga (2011).
} 
Theorem 2 A cost sharing solution $\overline{W S}^{\alpha}$ satisfies

i) Demand Ranking and Fair Rent if and only if $y=y^{0}$.

ii) Demand Monotonicity if and only if $y=y^{K}$.

iii) Fair Rent and Positive Rent if and only if $y=y^{1}$.

Proof. Consider the cost matrix $c \in \Gamma(\{1,2,3\})$ such that $c_{01}=c_{02}=0$ and $c_{e}=1$ else. Let $c^{i}$ be the matrix in which we change the cost of $(i, 3)$ to zero and $c^{1,2}$ the cost matrix in which edges $(1,3)$ and $(2,3)$ both have a cost of zero. The shares for these cost matrices are given in Appendix, for a generic $\alpha$ (the parameter in CTUD) and for $N^{t}=\{1,2\}$ and $\{1\}$.

Consider $c^{1,2}$. We have that $\overline{W S}_{1}^{\alpha}\left((\{2,3\},\{1\}), c^{1,2}\right)=-\frac{\alpha(1-\alpha)}{2-\alpha}$ and $\overline{W S_{2}^{\alpha}}\left((\{2,3\},\{1\}), c^{1,2}\right)=$ $-\frac{\alpha}{2(2-\alpha)}$. Notice that 1 and 2 have the same cost to connect to the source and to agent 3 . We can thus apply Demand Ranking, which imposes that $\overline{W S}_{1}^{\alpha}\left((\{2,3\},\{1,2\}), c^{1,2}\right) \leq \overline{W S}_{2}^{\alpha}\left((\{2,3\},\{1\}), c^{1,2}\right)$. Demand Ranking is thus satisfied in that case if $\alpha \in\left[0, \frac{1}{2}\right]$.

By Fair Rent, we must have that $\overline{W S}_{1}^{\alpha}\left(\left(\{2,3\},\{1\}, c^{1,2}\right) \geq 0\right.$, (as agents 2 and 3 can connect independently at a cost of zero). Fair Rent is thus satisfied in that case if $\alpha \in\{0,1\}$.

Consider $c^{2}$. We have that $\overline{W S_{2}^{\alpha}}\left((\{2,3\},\{1\}), c^{2}\right)=-\frac{1}{2}$ and that $\overline{W S_{2}^{\alpha}}\left((\{3\},\{1,2\}), c^{2}\right)=-\alpha$. Demand Monotonicity imposes that $\overline{W S_{2}^{\alpha}}\left((\{3\},\{1,2\}), c^{2}\right) \leq \overline{W S}_{2}^{\alpha}\left((\{2,3\},\{1\}), c^{2}\right)$, or $\alpha \geq \frac{1}{2}$.

Consider $c^{\prime} \in \Gamma(\{1,2,3,4\})$ such that $c_{04}^{\prime}=1$ and $c_{e}^{\prime}=0$ else. Consider the problem $\left((\{2,3,4\},\{1\}), c^{\prime}\right)$. If agent 4 comes first and agent 1 just after him, the imputation of agent 1 is -1 . In all other permutations, the imputation of agent 1 is zero. Therefore, we need only to find the probabilities of permutations $(4,1,2,3)$ and $(4,1,3,2)$. These probabilities are

$$
\frac{1-\alpha}{3-2 \alpha} \frac{1-\alpha}{2-\alpha} \alpha
$$

Thus, $\overline{W S_{1}^{\alpha}}\left(\left(\{2,3,4\},\{1\}, c^{\prime}\right)=\frac{-2 \alpha(1-\alpha)^{2}}{(3-2 \alpha)(2-\alpha)}\right.$. Suppose now that agent 1 wants to be connected. The problem becomes $\left((\{1,2,3,4\},\{\emptyset\}), c^{\prime}\right)$. The probabilities of permutations $(4,1,2,3)$ and $(4,1,3,2)$ become

$$
\frac{1}{4} \frac{1}{3} \frac{1}{2}=\frac{1}{24}
$$

and $\overline{W S}_{1}^{\alpha}\left((\{1,2,3,4\},\{\emptyset\}), c^{\prime}\right)=-\frac{1}{12}$. By Demand Monotonicity, we must have that $\overline{W S}_{1}^{\alpha}\left(\left(\{2,3,4\},\{1\}, c^{\prime}\right) \leq\right.$ $\overline{W S}_{1}^{\alpha}\left((\{1,2,3,4\},\{\emptyset\}), c^{\prime}\right)$, or

$$
\frac{-2 \alpha(1-\alpha)^{2}}{(3-2 \alpha)(2-\alpha)} \leq-\frac{1}{12}
$$

Within the interval $[0,1]$, the inequality is satisfied when $\alpha \in\left[\frac{19-\sqrt{73}}{24}, \frac{1}{2}\right]$.

i) The restrictions imposed by Demand Ranking and Fair Rent leave us with $\overline{W S}^{0}=y^{0}$ as our only candidate. By Lemma $2, y^{0}$ satisfies all properties.

ii) The restrictions imposed by Demand Monotonicity leave us with $\overline{W S^{\frac{1}{2}}}=y^{K}$. By Lemma $2, y^{K}$ satisfies all properties.

iii) The restrictions imposed by Fair Rent leaves us $\overline{W S}^{0}=y^{0}$ and $\overline{W S}^{1}=y^{1}$ as our only candidates. By Lemma 2, $y^{0}$ fails Positive Rent while $y^{1}$ satisfies it. $y^{1}$ also satisfies the other properties.

The previous theorem allows for interesting contrasts between the solutions. If we fear manipulations and require Demand Monotonicity, $y^{K}$ is the only solution. This should come as no surprise, as $y^{K}$, because it satisfies Equal Treatment, makes no distinction between demanders and non-demanders and simply looks at the cost they generate. If we put the accent on a stability property like Fair Rent but still want to remunerate non-demanders, $y^{1}$ is the only choice. We obtain $y^{0}$ by combining an incentive property (Demand Ranking) and a stability property (Fair Rent). This result can be seen as the impossibility of combining Fair Rent, Demand Ranking and Positive Rent.

Combining the results of Theorems 1 and 2, we obtain a characterization of $y^{K}$ with the properties of Group Independence, ETED, CTUD and Demand Monotonicity. 


\section{$5 \quad$ Linear network, shortest path and Steiner problems}

The case in which $N^{d}=N$ is the well-known minimum cost spanning tree model. It turns out that at the opposite end of the spectrum, when $\left|N^{d}\right|=1$, we have another well-known model. In that case, we only need to connect one agent to the source, with the (potential) help of other members of $N$. In our model, it means that we need to find the cheapest path from the source to $i$. The problem thus becomes a shortest path problem, solvable in polynomial time (Dijkstra (1959)). As for the mcst problem, the corresponding stand-alone cost game always has a non-empty core.

We can also see that it is equivalent to a much more general problem: suppose that agents in $N$ have a demand $q \in \mathbb{R}_{+}^{N}$. They need to be connected to the source to obtain these units, which have to be transported from the source to the nodes of these agents. We still have the cost matrix $c$, but now the cost to send $x$ units of goods on edge $(i, j)$ is $c_{i j} x$. A linear network problem is $(N, c, q)$, the set of agents, the cost matrix and the demand vector. It is easy to see that it is also a shortest path problem. For each agent $i$, we find the shortest path connecting him to the source. The optimal network configuration for $N$ is obtained by combining the individual shortest paths. See Trudeau (2009b) and Rosenthal (2013) for a discussion of this case. Since the network can be built in a decentralized manner, it seems very natural to impose that cost shares could also be decentralized and that $y(N, c, q)=\sum_{j \in N} y\left(N, c, e^{j}\right) q_{j}$, where $e^{j} \in \mathbb{R}_{+}^{N}$ is such that $e_{k}^{j}=1$ if $j=k$ and $e_{k}^{j}=0$ otherwise. Therefore, assigning cost shares to the whole linear network problem can be done by assigning cost shares for individual units of demand and summing up. But then, the linear network problem $\left(N, c, e^{j}\right)$, which consists in finding the shortest path between the source and $j$ to send him one unit of demand, is identical to the mcst problem with indifferent agents $((\{j\}, N \backslash\{j\}), c)$, where we need to build a mcst from the source to $j$.

It is worth noting that in that case, $y^{K}$ corresponds to the Shapley value studied in Rosenthal (2013). The linear network model is also very similar to the model in Trudeau (2009a), which is a general technological cooperation model with constant returns to scale. The only difference is that there is no underlying network structure. For cases in which we do not want to reward demanders (for instance if it generates pollution), a method is proposed that starts by assigning each agent its stand-alone cost before giving rebates related to the savings generated by its technology. It corresponds to $y^{1}$ for linear network problems. For cases such that $\left|N^{d}\right|>1, y^{1}$ is a very natural extension of the solution of Trudeau (2009a) that deals with economies of scale.

As mentioned in the introduction, we can view the minimum cost Steiner tree problem of Bergantinos et al. (2011) as our model with the added constraint that $y_{i}=0$ for all $i \in N^{t}$. If we add to this constraint Group Independence and ETED, we characterize $y^{0}$, which is nothing but the Shapley value for such problems.

\section{References}

Bahel, E., Trudeau, C., 2013. A discrete cost sharing model with technological cooperation. Int. J. Game Theory 42, 439-460.

Bergantinos, G., Lorenzo, L., Lorenzo-Freire, S., Vidal-Puga, J., 2011. Minimum cost Steiner tree problems. mimeo, University of Vigo.

Bergantinos, G., Vidal-Puga, J., 2007. A fair rule in minimum cost spanning tree problems. J. Econ. Theory 137, 326-352.

Bird, C., 1976. On cost allocation for a spanning tree: A game theoretic approach. Networks 6, 335-350.

Dijkstra, E. W., 1959. A note on two problems in connexion with graphs. Numerishe Mathematik 1 (1), 269-271. 
Feltkamp, V., Tijs, S., Muto, S., 1994. On the Irreducible Core and the Equal Remaining Obligations Rule of Minimum Cost Spanning Extension Problems. Tilburg University CentER Discussion Paper 94106.

Friedman, E., Moulin, H., 1999. Three methods to share joint costs or surplus. J. Econ. Theory 87, $275-312$.

Gomez-Rua, M., Vidal-Puga, J., 2011. Merge-proofness in minimum cost spanning tree problems. Int. J. Game Theory 40, 309-329.

Hwang, F. K., Richards, D. S., 1992. Steiner tree problems. Networks 22, 55-89.

Kalai, E., Samet, D., 1987. On weighted Shapley values. Int. J. Game Theory 16, 205-222.

Kar, A., 2002. Axiomatization of the Shapley value on minimum cost spanning tree games. Games Econ. Behav. 38, 265-277.

Moulin, H., 1995. On additive methods to share joint costs. Jpn. Econ. Rev. 46, 303-332.

Moulin, H., Sprumont, Y., 2006. Responsibility and cross-subsidization in cost sharing. Games Econ. Behav. 55, 152-188.

Rosenthal, E. C., 2013. Shortest path games. Eur. J. Oper. Res. 224 (1), $132-140$.

Trudeau, C., 2009a. Cost sharing with multiple technologies. Games Econ. Behav. 67, 695-707.

Trudeau, C., 2009b. Network flow problems and permutationally concave games. Math. Soc. Sci. 58, $121-131$.

Trudeau, C., 2012. A new stable and more responsive cost sharing solution for mcst problems. Games Econ. Behav. 75, 402-412.

\section{A Appendix}

\section{A.1 Proof of Lemma 1}

Proof. The proof is by induction.

Suppose that $m=2$. Then, $\operatorname{det}(M)=M_{11}-M_{12}>0$, since $M_{11}>0$ and $M_{12} \leq 0$.

Suppose that we have shown that $\operatorname{det}(M)>0$ whenever the dimension is not larger than $k$. We show that it implies that $\operatorname{det}(M)>0$ whenever the dimension is $k+1$.

Suppose that $m=k+1$. To compute the determinant, decompose the matrix along line $k$. By definition, $M_{k l}=0$ for all $l<k$. Thus, $\operatorname{det}(M)=M_{k k} \operatorname{det}\left(M^{k k}\right)-M_{k, k+1} \operatorname{det}\left(M^{k, k+1}\right)$, where $M^{i j}$ is the matrix $M$ to which we have removed the $i^{t h}$ line and $j^{t h}$ column. By definition, both $M^{k k}$ and $M^{k, k+1}$ are in $\Psi$. Since they are of dimension $k$, by the induction $\operatorname{argument,} \operatorname{det}\left(M^{k k}\right)>0$ and $\operatorname{det}\left(M^{k, k+1}\right)>0$. Since $M_{k k}>0$ and $M_{k, k+1} \leq 0$, we have that $\operatorname{det}(M)>0$.

\section{A.2 Weighted Shapley values $W S^{\alpha}$ satisfy the properties of Theorem 1}

Lemma 3 The weighted Shapley value $W S^{\alpha}$ satisfies Group Independence, Equal Treatment for Equal Demanders and Consistent Treatment for Unequal Demanders, with $\alpha \in(0,1)$ the parameter in CTUD.

Proof. Suppose that for any $i \in S, j \in N \backslash S, c_{i j} \geq \max \left(c_{0 i}, c_{0 j}\right)$. Let $\pi \in \Pi(N)$ and $\pi^{\prime} \in \Pi(S)$ be such that $B\left(i, \pi^{\prime}\right)=B(i, \pi) \cap S$. We have that $C\left(B(i, \pi) \cup\{i\},\left(N^{d}, N^{t}\right), c\right)-C\left(B(i, \pi),\left(N^{d}, N^{t}\right), c\right)=$ $\left.C\left(B\left(i, \pi^{\prime}\right) \cup\{i\},\left(N^{d}, N^{t}\right), c^{S}\right)-C\left(B(i, \pi), N^{d} \cap S, N^{t} \cap S\right), c^{S}\right)$. Therefore, the presence of agents in $N \backslash S$ do not affect the shares of agents in $S$ and thus $W S^{\alpha}$ satisfies Group Independence. 
Suppose that the cost matrices $c, c^{\prime} \in \Gamma(N)$ are such that $c_{i j}^{\prime}<c_{i j}$ and $c_{e}^{\prime}=c_{e}$ else. Notice that $C\left(S \cup\{i, j\},\left(N^{d}, N^{t}\right), c^{\prime}\right) \leq C\left(S \cup\{i, j\},\left(N^{d}, N^{t}\right), c\right)$ and $C\left(S,\left(N^{d}, N^{t}\right), c^{\prime}\right)=C\left(S,\left(N^{d}, N^{t}\right), c\right)$ for all $S \subseteq N \backslash\{i, j\}$.

For $S \subseteq N \backslash\{i, j\}$, notice that $C(S \cup\{i, j\})$ appears in $W S_{i}^{\alpha}$ only when associated to the permutations $\left(\pi^{\prime}, i, \pi\right)$, where $\pi^{\prime} \in \Pi(S \cup j)$ and $\pi \in \Pi(N \backslash(S \cup\{i, j\}))$, i.e. to permutations of $N$ where the first $|S|+1$ agents are the agents in $S \cup\{j\}$, the $(|S|+1)^{t h}$ player is agent $i$, with the remaining players in $N \backslash(S \cup\{i, j\})$. Similarly, $C(S \cup\{i, j\})$ affects agent $j$ only when associated to the permutations $\left(\pi^{\prime \prime}, j, \pi\right)$, where $\pi^{\prime \prime} \in \Pi(S \cup i)$ and $\pi \in \Pi(N \backslash(S \cup\{i, j\}))$.

We can write $W S_{i}^{\alpha}=\sum_{S \subseteq N} \phi_{i}(S) C(S)$, where $\phi_{i}(S)$ is the weight of $C(S)$ in the weighted Shapley value formula for agent $i$. By the above discussion we have that

$$
\phi_{i}(S \cup\{i, j\})=\sum_{\pi^{\prime} \in \Pi(S \cup j)} \sum_{\pi \in \Pi(N \backslash(S \cup\{i, j\}))} p\left(\left(\pi^{\prime}, i, \pi\right)\right)
$$

By the definition of $p(\cdot)$, we have, for a fixed $\pi \in \Pi(N \backslash(S \cup\{i, j\}))$, that

$$
\sum_{\pi^{\prime} \in \Pi(S \cup j)} p\left(\left(\pi^{\prime}, i, \pi\right)\right)=p(i, \pi)
$$

where $p(i, \pi)$ is the probability of permutation $(i, \pi) \in \Pi(N \backslash(S \cup\{j\}))$. Thus,

$$
\begin{aligned}
\phi_{i}(S \cup\{i, j\}) & =\sum_{\pi \in \Pi(N \backslash(S \cup\{i, j\}))} p((i, \pi)) \\
& =\sum_{\pi \in \Pi(N \backslash(S \cup\{i, j\}))} \prod_{k=0}^{n-|S|-3} \frac{w_{\pi_{n-k}}}{(1-\alpha) D+\alpha T-\sum_{l=1}^{k-1} w_{l}} \frac{w_{i}}{(1-\alpha) D^{S \cup\{i, j\}}+\alpha T^{S \cup\{i, j\}}} \\
& =\frac{w_{i}}{(1-\alpha) D^{S \cup\{i, j\}}+\alpha T^{S \cup\{i, j\}}} \sum_{\pi \in \Pi(N \backslash(S \cup\{i, j\}))} \prod_{k=0}^{n-|S|-3} \frac{w_{\pi_{n-k}}}{(1-\alpha) D+\alpha T-\sum_{l=1}^{k-1} w_{l}}
\end{aligned}
$$

where $D^{S}=\left|N^{d} \cap S\right|$ and $T^{S}=\left|N^{t} \cap S\right|$ are the number of demanders and non-demanders in $S$. We have that $D=D^{N}$ and $T=T^{N}$.

In the same manner, we have that

$$
\phi_{j}(S \cup\{i, j\})=\frac{w_{j}}{(1-\alpha) D^{S \cup\{i, j\}}+\alpha T^{S \cup\{i, j\}}} \sum_{\pi \in \Pi(N \backslash(S \cup\{i, j\}))} \prod_{k=0}^{n-|S|-3} \frac{w_{\pi_{n-k}}}{(1-\alpha) D+\alpha T-\sum_{l=1}^{k-1} w_{l}} .
$$

Let $\Delta C(S)=C\left(S,\left(N^{d}, N^{t}\right), c^{\prime}\right)-C\left(S,\left(N^{d}, N^{t}\right), c\right)$. Given that $C\left(S \cup\{i, j\},\left(N^{d}, N^{t}\right), c^{\prime}\right) \leq C(S \cup$ $\left.\{i, j\},\left(N^{d}, N^{t}\right), c\right)$ for all $S \subseteq N \backslash\{i, j\}$ and $C\left(R,\left(N^{d}, N^{t}\right), c^{\prime}\right)=C\left(R,\left(N^{d}, N^{t}\right), c\right)$ otherwise, we have, for $k \in\{i, j\}$, that

$$
y_{k}\left(\left(N^{d}, N^{t}\right), c^{\prime}\right)-y_{k}\left(\left(N^{d}, N^{t}\right), c\right)=w_{k} \sum_{S \subseteq N \backslash\{i, j\}} A(S) \Delta C(S \cup\{i, j\})
$$

where

$$
A(S)=\frac{1}{(1-\alpha) D^{S \cup\{i, j\}}+\alpha T^{S \cup\{i, j\}}} \sum_{\pi \in \Pi(N \backslash(S \cup\{i, j\}))} \prod_{k=0}^{n-|S|-3} \frac{w_{\pi_{n-k}}}{(1-\alpha) D+\alpha T-\sum_{l=1}^{k-1} w_{l}}
$$

Therefore, we have that

$$
\frac{y_{i}\left(\left(N^{d}, N^{t}\right), c^{\prime}\right)-y_{i}\left(\left(N^{d}, N^{t}\right), c\right)}{y_{j}\left(\left(N^{d}, N^{t}\right), c^{\prime}\right)-y_{j}\left(\left(N^{d}, N^{t}\right), c\right)}=\frac{w_{i}}{w_{j}}
$$


If $i, j \in N^{d}$ or $i, j \in N^{t}, w_{i}=w_{j}$ and thus

$$
y_{i}\left(\left(N^{d}, N^{t}\right), c^{\prime}\right)-y_{i}\left(\left(N^{d}, N^{t}\right), c\right)=y_{j}\left(\left(N^{d}, N^{t}\right), c^{\prime}\right)-y_{j}\left(\left(N^{d}, N^{t}\right), c\right) .
$$

ETED is satisfied. If $i \in N^{d}$ and $j \in N^{t}, w_{i}=(1-\alpha)$ and $w_{j}=\alpha$. Thus,

$$
\alpha\left[y_{i}\left(\left(N^{d}, N^{t}\right), c^{\prime}\right)-y_{i}\left(\left(N^{d}, N^{t}\right), c\right)\right]=(1-\alpha)\left[y_{j}\left(\left(N^{d}, N^{t}\right), c^{\prime}\right)-y_{j}\left(\left(N^{d}, N^{t}\right), c\right)\right]
$$

and CTUD is satisfied.

\section{A.3 Independence of the properties of Theorem 1}

Lemma 4 The properties of Theorem 1 are independent.

Proof. We need to show that as soon as we remove one of the three properties in Theorem 1 (Group Independence, Equal Treatment for Equal Demanders and Consistent Treatment for Unequal Demanders), another solution appears.

Group Independence: Group Independence is needed in the proof of Theorem 1 to define values when we do not have any relevant paths. In such a case, define $y_{i}\left(\left(N^{d}, N^{t}\right), c\right)=\frac{C\left(N,\left(N^{d}, N^{t}\right), c\right)}{|N|}$ for all $i \in N$. Follow the method described in the proof of Theorem 1 to define a cost sharing solution that satisfies ETED and CTUD but fails Group Independence.

Equal Treatment for Equal Demanders: Proceed as in the proof of Theorem 1, with the following modification. If we remove a relevant edge between $i$ and $j$, with $i, j \in N^{d}$ or $i, j \in N^{t}$, use the following equality instead to obtain the relevant equation between $y_{i}(c)-y_{i}\left(c^{i j}\right)$ and $y_{j}(c)-y_{j}\left(c^{i j}\right)$ :

$$
\left(1+c_{0 i} c_{0 j}^{2}\right)\left[y_{i}\left(\left(N^{d}, N^{t}\right), c^{\prime}\right)-y_{i}\left(\left(N^{d}, N^{t}\right), c\right)\right]=\left(1+c_{0 i}^{2} c_{0 j}\right)\left[y_{j}\left(\left(N^{d}, N^{t}\right), c^{\prime}\right)-y_{j}\left(\left(N^{d}, N^{t}\right), c\right)\right]
$$

and follow the method described in the proof of Theorem 1. Notice that $1+c_{0 i} c_{0 j}^{2}>0$ and $1+c_{0 i}^{2} c_{0 j}>0$, assuring that the resulting matrix is still in $\Psi$. It defines a cost sharing solution that satisfies Group Independence and CTUD but fails ETED.

Consistent Treatment for Unequal Demanders: Proceed as in the proof of Theorem 1, with the following modification. If we remove a relevant edge between $i$ and $j$, with $i \in N^{d}$ and $j \in N^{t}$ use the following equality instead to obtain the relevant equation between $y_{i}(c)-y_{i}\left(c^{i j}\right)$ and $y_{j}(c)-y_{j}\left(c^{i j}\right)$ :

$$
\begin{aligned}
y_{i}^{c}\left(\left(N^{d}, N^{t}\right), c^{\prime}\right)-y_{i}^{c}\left(\left(N^{d}, N^{t}\right), c\right) & =y_{j}^{c}\left(\left(N^{d}, N^{t}\right), c^{\prime}\right)-y_{j}^{c}\left(\left(N^{d}, N^{t}\right), c\right) \text { if } \min \left(c_{0 i}, c_{0 j}\right)=0 \\
\frac{1}{3}\left[y_{i}^{c}\left(\left(N^{d}, N^{t}\right), c^{\prime}\right)-y_{i}^{c}\left(\left(N^{d}, N^{t}\right), c\right)\right] & =\frac{2}{3}\left[y_{j}^{c}\left(\left(N^{d}, N^{t}\right), c^{\prime}\right)-y_{j}^{c}\left(\left(N^{d}, N^{t}\right), c\right)\right] \text { if } \min \left(c_{0 i}, c_{0 j}\right)>0
\end{aligned}
$$

and follow the method described in the proof of Theorem 1. Notice that the resulting matrix is still in $\Psi$. It defines a cost sharing solution that satisfies Group Independence and ETED but fails CTUD (for any value of $\alpha$ ).

\section{A.4 $y^{0}$ and $y^{1}$ satisfy the properties of Theorem 1}

Lemma 5 i) $y^{0}$ satisfies Group Independence and Equal Treatment for Equal Demanders. It satisfies Consistent Treatment for Unequal Demanders, with $\alpha$ the parameter in that property, equal to zero.

ii) $y^{1}$ satisfies Group Independence and Equal Treatment for Equal Demanders. It satisfies Consistent Treatment for Unequal Demanders, with $\alpha$ the parameter in that property, equal to one.

Proof. i) We show that $y^{0}$ satisfies Group Independence, Equal Treatment for Equal Demanders and Consistent Treatment for Unequal Demanders for $\alpha=0$.

Suppose that for all $i \in S, j \in N \backslash S$, there are no relevant edges. Then, $\bar{C}\left((R \cup T) \cap N^{d},\left(N^{d}, N^{t}\right), c\right)=$ $\bar{C}\left(R \cap N^{d},\left(N^{d}, N^{t}\right), c\right)+\bar{C}\left(T \cap N^{d},\left(N^{d}, N^{t}\right), c\right)$ for all $R \subseteq S$ and $T \subseteq N \backslash S$. By the properties of the Shapley value, $S h_{i}\left(\bar{C}\left(\cdot,\left(N^{d}, N^{t}\right), c\right)\right)=S h_{i}\left(\bar{C}\left(\cdot,\left(N^{d} \cap S, N^{t} \cap S\right), c^{S}\right)\right)$ for all $i \in S \cap N^{d}$ and 
$S h_{j}\left(\bar{C}\left(\cdot,\left(N^{d}, N^{t}\right), c\right)\right)=S h_{j}\left(\bar{C}\left(\cdot,\left(N^{d} \backslash S, N^{t} \backslash S\right), c^{N \backslash S}\right)\right)$ for all $j \in N^{d} \backslash S$. Since $y_{i}^{0}\left(\left(N^{d}, N^{t}\right), c\right)=0$ for all $i \in N^{t}$ and all $c$, Group Independence is satisfied.

Let $c, c^{\prime}$ be such that for $i, j \in N^{d}, c_{i j}^{\prime}<c_{i j}$ and $c_{e}^{\prime}=c_{e}$ else. The edge $(i, j)$ can only be used by a coalition $S$ if $i, j \in S$. Therefore, $\bar{C}\left(T,\left(N^{d}, N^{t}\right), c^{\prime}\right)=\bar{C}\left(T,\left(N^{d}, N^{t}\right), c\right)$ for all $T \subseteq N$ such that $\{i, j\} \varsubsetneqq T$. By the properties of the Shapley value, $y_{i}^{0}\left(\left(N^{d}, N^{t}\right), c^{\prime}\right)-y_{i}^{0}\left(\left(N^{d}, N^{t}\right), c\right)=$ $y_{j}^{0}\left(\left(N^{d}, N^{t}\right), c^{\prime}\right)-y_{j}^{0}\left(\left(N^{d}, N^{t}\right), c\right)$. Since $y_{i}^{0}\left(\left(N^{d}, N^{t}\right), c\right)=0$ for all $i \in N^{t}$ and all $c$, the part of ETED that applies to agents in $N^{t}$ is satisfied trivially. Therefore, ETED is satisfied.

Let $c, c^{\prime}$ be such that for $i \in N^{d}$ and $j \in N^{t}, c_{i j}^{\prime}<c_{i j}$ and $c_{e}^{\prime}=c_{e}$ else. Since $y_{j}^{0}\left(\left(N^{d}, N^{t}\right), c\right)=$ $y_{j}^{0}\left(\left(N^{d}, N^{t}\right), c^{\prime}\right)=0$, CTUD is satisfied for $\alpha=0$.

ii) We show that $y^{1}$ satisfies Group Independence, Equal Treatment for Equal Demanders and Consistent Treatment for Unequal Demanders for $\alpha=1$.

Suppose that for all $i \in S, j \in N \backslash S$, there are no relevant edges. Then, $C^{N^{d}}\left((R \cup T) \cap N^{d},\left(N^{d}, N^{t}\right), c\right)=$ $C^{N^{d}}\left(R \cap N^{d},\left(N^{d}, N^{t}\right), c\right)+C^{N^{d}}\left(T \cap N^{d},\left(N^{d}, N^{t}\right), c\right)$ for all $R \subseteq S$ and $T \subseteq N \backslash S$. By the properties of the Shapley value, $S h_{i}\left(C^{N^{d}}\left(\cdot,\left(N^{d}, N^{t}\right), c\right)\right)=S h_{i}\left(C^{N^{d}}\left(\cdot,\left(N^{d} \cap S, N^{t} \cap S\right), c^{S}\right)\right)$ for all $i \in S \cap N^{d}$ and $S h_{j}\left(C^{N^{d}}\left(\cdot,\left(N^{d}, N^{t}\right), c\right)\right)=S h_{j}\left(C^{N^{d}}\left(\cdot,\left(N^{d} \backslash S, N^{t} \backslash S\right), c^{N \backslash S}\right)\right)$ for all $j \in N^{d} \backslash S$. We also have that $\tilde{C}\left((R \cup T) \cap N^{t},\left(N^{d}, N^{t}\right), c\right)=\tilde{C}\left(R \cap N^{t},\left(N^{d}, N^{t}\right), c\right)+\tilde{C}\left(T \cap N^{t},\left(N^{d}, N^{t}\right), c\right)$ for all $R \subseteq S$ and $T \subseteq N \backslash S$. By the properties of the Shapley value $S h_{i}\left(\tilde{C}\left(\cdot,\left(N^{d}, N^{t}\right), c\right)\right)=S h_{i}\left(\tilde{C}\left(\cdot,\left(N^{d} \cap S, N^{t} \cap S\right), c^{S}\right)\right)$ for all $i \in S \cap N^{t}$ and $S h_{j}\left(\tilde{C}\left(\cdot,\left(N^{d}, N^{t}\right), c\right)\right)=S h_{j}\left(\tilde{C}\left(\cdot,\left(N^{d} \cap T, N^{t} \cap T\right), c^{N \backslash S}\right)\right)$ for all $j \in N^{t} \backslash S$. Group Independence is satisfied.

Let $c, c^{\prime}$ be such that for $i, j \in N^{d}, c_{i j}^{\prime}<c_{i j}$ and $c_{e}^{\prime}=c_{e}$ else. The edge $(i, j)$ can only be used by a coalition $S$ if $i, j \in S$. Therefore, $C^{N^{d}}\left(T,\left(N^{d}, N^{t}\right), c^{\prime}\right)=C^{N^{d}}\left(T,\left(N^{d}, N^{t}\right), c\right)$ for all $T \subseteq N$ such that $\{i, j\} \varsubsetneqq T$. By the properties of the Shapley value, $y_{i}^{1}\left(\left(N^{d}, N^{t}\right), c^{\prime}\right)-y_{i}^{1}\left(\left(N^{d}, N^{t}\right), c\right)=$ $y_{j}^{1}\left(\left(N^{d}, N^{t}\right), c^{\prime}\right)-y_{j}^{1}\left(\left(N^{d}, N^{t}\right), c\right)$. Let $c, c^{\prime}$ be such that for $i, j \in N^{t}, c_{i j}^{\prime}<c_{i j}$ and $c_{e}^{\prime}=c_{e}$ else. The edge $(i, j)$ can only be used by a coalition $S$ if $i, j \in S$. Therefore, $\tilde{C}\left(T,\left(N^{d}, N^{t}\right), c^{\prime}\right)=$ $\tilde{C}\left(T,\left(N^{d}, N^{t}\right), c\right)$ for all $T \subseteq N$ such that $\{i, j\} \varsubsetneqq T$. By the properties of the Shapley value, $y_{i}^{1}\left(\left(N^{d}, N^{t}\right), c^{\prime}\right)-y_{i}^{1}\left(\left(N^{d}, N^{t}\right), c\right)=y_{j}^{1}\left(\left(N^{d}, N^{t}\right), c^{\prime}\right)-y_{j}^{1}\left(\left(N^{d}, N^{t}\right), c\right)$. Therefore, ETED is satisfied.

Let $c, c^{\prime}$ be such that for $i \in N^{d}$ and $j \in N^{t}, c_{i j}^{\prime}<c_{i j}$ and $c_{e}^{\prime}=c_{e}$ else. Since $C^{N^{d}}\left(S,\left(N^{d}, N^{t}\right), c\right)=$ $C^{N^{d}}\left(S,\left(N^{d}, N^{t}\right), c^{\prime}\right)$ for all $S \subseteq N^{d}$, we have that $y_{i}^{1}\left(\left(N^{d}, N^{t}\right), c\right)=y_{i}^{1}\left(\left(N^{d}, N^{t}\right), c^{\prime}\right)$. Therefore, CTUD is satisfied for $\alpha=1$.

\section{A.5 Proof of Lemma 2}

Proof. i) $y^{0}$ satisfies Demand Ranking and Fair Rent but fails Demand Monotonicity and Positive Rent.

Suppose that for $i \in N^{d}, j \in N^{t}, c_{i k}=c_{j k}$ for all $k \in N_{0} \backslash\{i, j\}$. By definition, we will thus have that $\bar{C}\left(S \cup\{i\},\left(N^{d}, N^{t}\right), c\right) \geq \bar{C}\left(S,\left(N^{d}, N^{t}\right), c\right)$ for all $S \subseteq N^{d} \backslash\{i\}$, as the benefits that agents in $S$ can obtain by connecting through agent $i$ 's location can already be obtained by connecting through agent $j$ 's location. By definition, $y_{i}^{0}\left(\left(N^{d}, N^{t}\right), c\right) \geq 0=y_{j}^{0}\left(\left(N^{d}, N^{t}\right), c\right)$. Therefore, Demand Ranking is satisfied.

Fair Rent is trivially satisfied as

$$
\sum_{i \in N^{d}} y_{i}^{0}\left(\left(N^{d}, N^{t}\right), c\right)=C\left(N,\left(N^{d}, N^{t}\right), c\right) \leq C\left(N^{d},\left(N^{d}, N^{t}\right), c\right) .
$$

It also implies that Positive Rent is not satisfied.

Consider $c^{2}$ described in Section A.6. We have that $y_{2}^{0}\left((\{3\},\{1,2\}), c^{2}\right)=0$ and $y_{2}^{0}\left((\{2,3\},\{1\}), c^{2}\right)=$ $-\frac{1}{2}$, violating Demand Monotonicity.

ii) $y^{K}$ satisfies Demand Monotonicity, Demand Ranking and Positive Rent but fails Fair Rent.

For $i \in N^{t}$, we have that $C\left(S \cup\{i\},\left(N^{d}, N^{t}\right), c\right) \leq C\left(S \cup\{i\},\left(N^{d} \cup\{i\}, N^{t} \backslash\{i\}\right), c\right)$ and $C\left(S,\left(N^{d}, N^{t}\right), c\right) \leq$ $C\left(S,\left(N^{d} \cup\{i\}, N^{t} \backslash\{i\}\right), c\right)$ for all $S \subseteq N \backslash\{i\}$. By the properties of the Shapley value, $y_{i}\left(\left(N^{d}, N^{t}\right), c\right) \leq$ $y_{i}\left(\left(N^{d} \cup\{i\}, N^{t} \backslash\{i\}\right), c\right)$. Demand Monotonicity is satisfied. 
Suppose that for $i \in N^{d}, j \in N^{t}, c_{i k}=c_{j k}$ for all $k \in N_{0} \backslash\{i, j\}$. We have that $C(S \cup$ $\left.\{i\},\left(N^{d}, N^{t}\right), c\right) \geq C\left(S \cup\{j\},\left(N^{d}, N^{t}\right), c\right)$ for all $S \subseteq N \backslash\{i, j\}$. By the properties of the Shapley value, $y_{i}\left(\left(N^{d}, N^{t}\right), c\right) \geq y_{j}\left(\left(N^{d}, N^{t}\right), c\right)$. Demand Ranking is satisfied.

Consider $c^{1,2}$ described in Section A.6. We have that $y_{1}^{K}\left((\{2,3\},\{1\}), c^{1,2}\right)=-\frac{1}{6}$. However, by Fair Rent, we should have that $y_{1}^{K}\left((\{2,3\},\{1\}), c^{1,2}\right)=0$ as agents 2 and 3 can connect independently at a cost of zero. Fair Rent is not satisfied. It also shows that Positive Rent is satisfied.

iii) $y^{1}$ satisfies Fair Rent and Positive Rent but fails Demand Monotonicity and Demand Ranking.

Fair Rent is trivially satisfied as

$$
\sum_{i \in N^{d}} y_{i}^{1}\left(\left(N^{d}, N^{t}\right), c\right)=C\left(N^{d},\left(N^{d}, N^{t}\right), c\right) .
$$

Consider $c^{1,2}$ described in Section A.6. We have that $y_{1}^{1}\left((\{2,3\},\{1\}), c^{1,2}\right)=0, y_{2}^{1}\left((\{2,3\},\{1\}), c^{1,2}\right)=$ $-\frac{1}{2}$. Demand Ranking is not satisfied

Consider a problem with $N=\{1,2,3,4\}$ and $c^{\prime}$ such that $c_{03}^{\prime}=c_{04}^{\prime}=1$ and $c_{e}^{\prime}=0$ for all other edges. We have that $y_{2}^{1}\left((\{3,4\},\{1,2\}), c^{\prime}\right)=-\frac{1}{2}$ and $y_{2}^{1}\left((\{2,3,4\},\{1\}), c^{\prime}\right)=-\frac{2}{3}$. The example shows that Demand Monotonicity is not satisfied but that Positive Rent is.

\section{A.6 Shares for $c, c^{1}, c^{2}$ and $c^{1,2}$ (from Theorem 2)}

We are interested in the shares for the cases in which $N^{t}=\{1,2\}$ and $N^{t}=\{1\}$. The following table gives the shares for a generic $\alpha$, the parameter in CTUD. A discussion follows on how they were obtained.

$$
\begin{array}{ccc}
d & y((\{3\},\{1,2\}), d) & y((\{2,3\},\{1\}), d) \\
c & (0,0,1) & (0,0,1) \\
c^{1} & (-\alpha, 0, \alpha) & (-\alpha, 0, \alpha) \\
c^{2} & (0,-\alpha, \alpha) & \left(0,-\frac{1}{2}, \frac{1}{2}\right) \\
c^{1,2} & \left(-\frac{\alpha^{2}}{\alpha+1},-\frac{\alpha^{2}}{\alpha+1}, \frac{2 \alpha^{2}}{\alpha+1}\right) & \left(-\frac{\alpha(1-\alpha)}{2-\alpha},-\frac{\alpha}{2(2-\alpha)}, \frac{\alpha(3-2 \alpha)}{2(2-\alpha)}\right)
\end{array}
$$

The cost shares for $c$ are trivially obtained by Group Independence.

For $c^{1}$, notice that agent 2 has no relevant edge to other agents, so we can look only at agents 1 and 3 . The permutation $(1,3)$ assigns zero to both agents, but the imputation is $(-1,1)$ for the permutation $(3,1)$. The probability of that permutation, by definition of weighted Shapley values, is $\alpha$.

The results are similar for $c^{2}$ : we can remove agent 1 by Group Independence, and the only nonzero imputation occurs for the permutation $(3,2)$. The probability of the permutation is $\alpha$ if $2 \in N^{t}$ and $\frac{1}{2}$ if $2 \in N^{d}$.

Finally, for $c^{1,2}$, the permutation $(3,1,2)$ yields an imputation of $(-1,0,1)$ while for the permutation $(3,2,1)$ it is $(0,-1,1)$. All other permutations give shares of zero to all agents. We need to compute $p(3,1,2)$ and $p(3,2,1)$. If $2 \in N^{t}$, we have that $p(3,1,2)=p(3,2,1)=\frac{\alpha}{2 \alpha+1-\alpha} \alpha=\frac{\alpha^{2}}{\alpha+1}$. If $2 \in N^{d}$, we have that $p(3,1,2)=\frac{1-\alpha}{\alpha+2(1-\alpha)} \alpha=\frac{\alpha(1-\alpha)}{2-\alpha}$ and $p(3,2,1)=\frac{\alpha}{\alpha+2(1-\alpha)} \frac{1-\alpha}{2(1-\alpha)}=\frac{\alpha}{2(2-\alpha)}$. 